\title{
Non-isotropic Outflows in the Infrared: ISO Imaging of LBVs
}

Norman R. Trams ${ }^{1}$, C.I. van Tuyll ${ }^{2}$, Robert H.M. Voors ${ }^{3}$, Alex de Koter ${ }^{2}$, Laurens B.F.M. Waters ${ }^{2}$, and Patrick W. Morris ${ }^{4}$

${ }^{1}$ Astrophysics Division, ESA Space Science Department, ESTEC, PO Box 299, 2200 AG Noordwijk, The Netherlands

2 Astronomical Institute, University of Amsterdam, Kruislaan 403, NL-1098 SJ Amsterdam, The Netherlands

${ }^{3}$ Astronomical Institute Utrecht, Princetonplein 5, Utrecht, The Netherlands

${ }^{4}$ SRON Laboratory for Space Research, Sorbonnelaan 2, NL-3584 CA, Utrecht, The Netherlands

\begin{abstract}
In this review we present the ISO $^{1}$ imaging of nebulae around Luminous Blue Variables. Three LBVs have been imaged with ISO: HR Car, AG Car and the LBV candidate G79.29+0.46. The ISOCAM instrument did not resolve the nebula around HR Car. However some nebular emission lines are seen in the spectral energy distribution of this source. A proper deconvolution of the images may resolve the nebula. For AG Car and G79.29+0.46 the nebula is clearly resolved. The structure and intensity distribution of the nebular emission is dependent on the wavelength, indicating a separation between the ionised matter and the dust in the nebulae. Some model calculations are presented for the G79.29+0.46 nebula.
\end{abstract}

\section{Introduction}

\subsection{LBV nebulae}

Most Luminous Blue Variables (LBVs) are surrounded by nebulae (see Smith, 1994 and Nota and Clampin, 1997 for reviews). These nebulae are thought to be the result of the interaction between the strong LBV wind and a surrounding medium that is due to an older mass loss phase of the star. Most of the galactic LBV nebulae have been discovered using optical imaging. For the more distant LBVs (e.g. in the LMC) the presence of the nebulae has been inferred from optical spectroscopy. In some cases these nebulae have been confirmed by Hubble Space Telescope images.

Using optical imaging in emission lines $(\mathrm{H} \alpha)$ the mass of the ionized matter in the nebula can be determined. The ionized masses are typically a few solar masses (Nota and Clampin, 1997). Most nebulae display some degree

${ }^{1}$ Based on observations with the Infrared Space Observatory (ISO). ISO is an ESA project with instruments funded by the ESA member states (especially the PI countries: France, Germany, The Netherlands and the United Kingdom) and with the participation of ISAS and NASA 
of bipolarity, which strengthens the interacting wind model for the formation of the nebulae. In this model the correlation between nebular mass and age points to the formation in subsequent mass loss episodes. Abundance studies of the nebula (Smith et al., 1997) and studies of the dust content of the nebulae (Waters et al., 1998) indicate that they are expelled in a Red Supergiant (RSG) phase. In that case the interacting wind model would point to interactions between the current LBV wind and the remnant of the RSG wind.

The dust in LBV nebulae has been discussed by Hutsemékers (1997) and Waters et al. (1998). The dust masses seen in these nebulae range from 0.0003 to about 0.04 solar masses. Hutsemékers (1997) finds a correlation between stellar luminosity and nebular dust mass. Large grains seem to dominate in the nebulae, and give rise to the observed dust scattering in AG Car and HD168625 (Viotti et al., 1988 and Hutsemékers et al., 1994). Waters et al. (1998) find evidence for crystalline silicates in a number of LBV nebulae from ISO SWS spectra (see also Waters et al., this volume).

\subsection{IR imaging of LBV nebulae}

Not many imaging observations in the infrared spectral region have been published. Most IR imaging has been done at near-infrared wavelengths $(2.2 \mu \mathrm{m}$, e.g. McGregor et al., 1988; Hutsemékers et al., 1994). At these wavelengths the nebular emission is mostly due to scattering of stellar radiation by the dust grains in the nebula. Only at longer wavelengths beyond 10 micron direct imaging of the nebular dust content is possible, since here the thermal continuum of the dust starts to dominate (e.g. Humphreys and Davidson, 1994).

Most LBVs in the galaxy and in the Magellanic Clouds have been detected by IRAS, however the spatial resolution of that instrument was too low to resolve the nebula. For AG Car McGregor et al. (1988) find extended emission from the nebula at 50 and $100 \mu \mathrm{m}$ using the KAO. For the LBV candidate G79.29+0.46 (Higgs et al., 1994) IRAS maps at 12, 25 and 60 micron resolving the nebula were published by Waters et al. (1996). Recently some LBVs have been imaged using ground based instrumentation. Voors et al. (1997) discuss TIMMI images of HR Car at 10 and $12.8 \mu \mathrm{m}$. Their images show a small (15") clumpy nebula. The most prominent features seen in the 10 micron image are an arclike structure about 3" SE of the star and a bright blob about 1.5" NW of the star. Interestingly enough the ionized matter seen in this image and the optical images (Clampin et al., 1995) show that the dust and ionized gas close to the star is more clumpy than the gas distribution further away. Voors et al. estimate the age of the inner nebula to be around 850 years.

Robberto and Herbst (1998) in a recent paper show IR imaging of the LBV HD168625 at 2.16, 4.7, 10, 11.6 and $20 \mu \mathrm{m}$ using a new instrument on UKIRT (MAX). These high resolution images (pixel size of 0.265 by 0.265 
arcsec) clearly resolve the nebula around this star at both wavelengths. They conlude that the IR emission of this nebula comes from a geometrically thin layer of dust on the outer edges of the lobes seen in the nebula of this star. They also conclude that the dust in the nebula around this star consists of large grains.

Unfortunately all ground based imaging observations of these stars at longer wavelengths are hampered by the presence of the earths atmosphere making the observations of these sometimes faint nebulae very time consuming.

\subsection{ISO imaging observations of LBVs}

The Infrared Space Observatory (ISO) was an ESA satellite launched in November 1995 . It consisted of a $60^{\prime} \mathrm{cm}$ superfluid helium cooled telescope and a complement of 4 spectroscopic and photometric instruments for the wavelength range from 2 to $200 \mu \mathrm{m}$ (see Kessler et al., 1996). The satellite worked for more than 2 years, and was finally switched off after the helium ran out in May 1998. Two ISO instruments were used for imaging: The ISOCAM instrument for the wavelength range 2 to $17 \mu \mathrm{m}$ with a pixel size of 1.5 to 6 arcsec (see Cesarski et al., 1996) and the ISOPHOT instrument for the longer wavelengths (20 to $200 \mu \mathrm{m}$ ) with a resolution of between 10 and 60 arcsec, using a raster scanning mode (see Lemke et al., 1996).

Only a few LBVs have been imaged using ISO. The main reason for this is that the resolution of the instruments was not very high (the diffraction pattern of the telescope was about $8 \operatorname{arcsec}$ wide at $10 \mu \mathrm{m}$ ), so only the largest nebula could be resolved, and also the sensitivity of especially the ISOCAM instrument was so high that the central star of many of these nebulae would completely saturate the detectors, since no coronographic mode was available. Only HR Car, AG Car and G79.29+0.46 have been imaged using ISOCAM. For those observations the Circular Variable Filters (CVFs) of ISOCAM were used as narrow band filters to minimise the risk of saturation by the central star. For G79.29+0.46 mapping using the ISOPHOT instrument was performed as well at longer wavelengths by Wendker and Molthagen (1998).

\section{Discussion on individual objects}

\subsection{HR Car}

The observations of HR Car were performed using ISOCAM, with the CVF set at 8 wavelenghts $(8.689 \mu \mathrm{m}, 8.993 \mu \mathrm{m}$ (Ar III), $11.480 \mu \mathrm{m}, 12.410 \mu \mathrm{m}$, $12.820 \mu \mathrm{m}$ (Ne II), $13.530 \mu \mathrm{m}, 15.580 \mu \mathrm{m}$ (Ne III) and $15.960 \mu \mathrm{m}$ ). Staring mode was used with a 6 arcsec pixel field of view, giving a total field of 180 by 180 arcsec. The observations were performed on February 19, 1996. 
The data were processed using the CAM Interactive Analysis $\left(\mathrm{CIA}^{2}\right)$. The nebula is not clearly resolved, although there is a hint for non sphericity towards the SE of the star (corresponding to the arc observed by Voors et al. in the TIMMI images). A spectral energy distibution of the central source and the surrounding area however clearly shows the nebular [NeII] emission line at $12.82 \mu \mathrm{m}$, indicating that the nebula is seen in the images. Also the continuum slope of the surrounding area is different compared to the continuum of the central source (see Trams et al., 1996). Unfortunately at this time no point spread function for the CVF of ISOCAM is available, therefore no deconvolution of the images can be performed to increase the resolution. A proper deconvolution may be able to resolve the nebula in this case.

\subsection{AG Car}

The observations of AG Car were performed using the same settings as the observations for HR Car. These observations were performed on February 17, 1996. Contour plots of the resulting images are given in Figure 1.
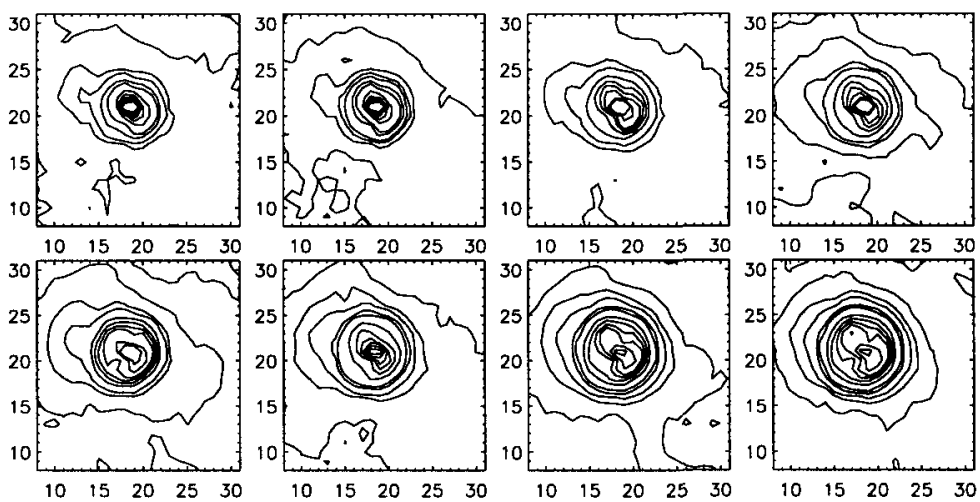

Fig. 1. Contour plots of the ISOCAM images of AG Car. North is to the top and East is on the left. Contours are for $0,5,10,20,30,40,50,75,100,200,300$, $400,500,750$ and $1000 \mathrm{mJy}$. The wavelengths of the images are from top left to bottom right: $8.689 \mu \mathrm{m}, 8.993 \mu \mathrm{m}, 11.480 \mu \mathrm{m}, 12.410 \mu \mathrm{m}, 12.820 \mu \mathrm{m}, 13.530 \mu \mathrm{m}$, $15.580 \mu \mathrm{m}$ and $15.960 \mu \mathrm{m}$.

The ring nebula is clearly seen in the CAM images. Also the bipolar structure that is clear in the optical images is found in the IR. The nebula

${ }^{2}$ CIA is a joint developement by the ESA Astrophysics Division and the ISOCAM consortium led by the ISOCAM PI, C. Cesarsky, Direction des Sciences de la Materie, C.E.A., France. 
is especially strong in the [Ne II] line at $12.82 \mu \mathrm{m}$. Interestingly the bipolar structure is strong in the continuum, i.e. in the thermal dust emission. Images of the [NeII] line emission (with the continuum subtracted) and the PAH emission band (the image at $11.48 \mu \mathrm{m}$ includes part of this band) show a clear difference in geometry. The ionized gas emission is more ring like, whereas the dust particles are concentrated in the bipolar structure. This is similar to the images shown by Nota et al. (1995), who show a clumpy bipolar nebula in the visual continuum and a smooth ring like structure in the $\mathrm{H} \alpha$ image.

\section{$2.3 \quad$ G79.29+0.46}

The nebula around the LBV candidate G79.29+0.46 is about 3 arcmin in diameter (Higgs et al., 1994). This is too large to fit in a single ISOCAM frame. We therefore observed this star using a 3 by 3 map with half a beam step size and 6 arcsec pixels. This gives a total field of view of 360 by 360 arcsec. The images were obtained at ten wavelengths. Apart from the ones mentioned above we also obtained images at 10 and at $17 \mu \mathrm{m}$. The images are shown as contour plots in Figure 2.
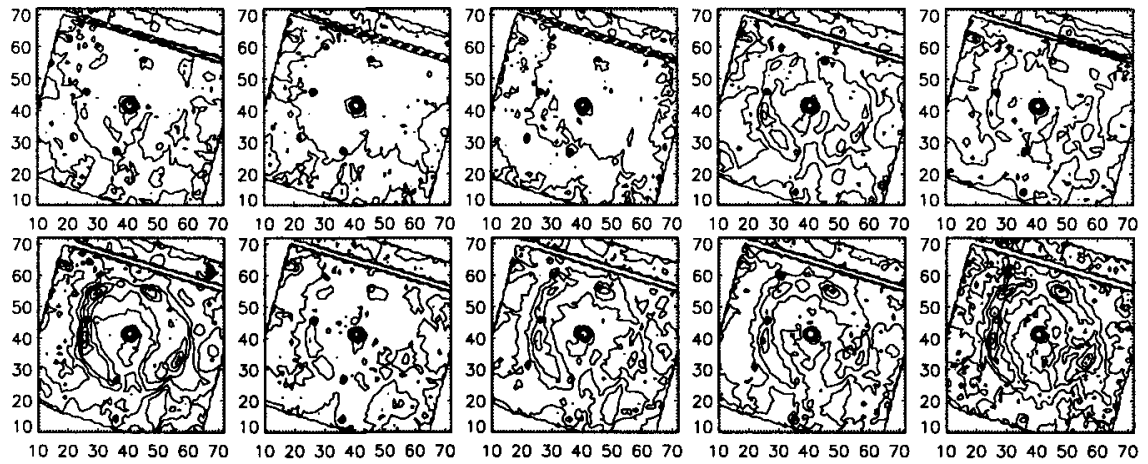

Fig. 2. Contour plots of the ISOCAM images of G79.29+0.46. North is to the top and East is to the left. Contours are for $0,10,20,30,40,50,75,100$, 200 and $500 \mathrm{mJy}$. The wavelengths of the images are from top left to bottom right: $8.689 \mu \mathrm{m}, 8.993 \mu \mathrm{m}, 10 \mu \mathrm{m}, 11.480 \mu \mathrm{m}, 12.410 \mu \mathrm{m}, 12.820 \mu \mathrm{m}, 13.530 \mu \mathrm{m}$, $15.580 \mu \mathrm{m}, 15.960 \mu \mathrm{m}$ and $17 \mu \mathrm{m}$.

The ring nebula is clearly seen in our ISOCAM images. Also some structure in the nebula is seen. The nebula is clumpy with very striking holes in the ring nebula on the north and southern side at all wavelengths. Since these holes are seen at all wavelenghts, they cannot be the result of an ionisation difference, but must be due to a difference in column density. Furthermore comparing the ISO images with larger IRAS maps of this area of the sky, the 
holes are seen towards the highest density in the Interstellar medium (the object is close to a molecular cloud that shows up as a clear rise in $60 \mu \mathrm{m}$ flux). This points to the fact that the nebula is probably the result of an interaction between (asymmetric) winds instead of an interaction between a wind and the local ISM.

The [Ne II] line emission image and that of the PAH emission were compared with the radio map presented by Higgs et al. (1994) and the $25 \mu \mathrm{m}$ map presented by Waters et al. (1996) and Wendker and Molthagen (1998). The [Ne II] emission coincides with the radio (free-free) emission and that the PAH emission coincides with the $25 \mu \mathrm{m}$ emission (dust). Where the [Ne II] image and the radio image show bright areas, the PAH and $25 \mu \mathrm{m}$ image show weaker emission. This is consistent with an ionisation of the nebula by the central star, since in that case in the dusty areas the UV radiation is shielded and therefore the ionisation of the nebula would be lower.

In the spectral energy distributions we calculated for this star, we can see that apart from the presence of the [Ne II] line in the nebula, there is also [Ne II] emission on the position of the central star. Furthermore the continuum at longer wavelengths is not stellar, but more nebular in shape (similar to HR Car). We therefore conclude that a small and unresolved nebula is present in G79.29+0.46 very close to the star (probably within 6-10 arcsec radius).

\section{A model for $\mathrm{G} 79.29+0.46$}

We have simulaneously modeled the central star G79.29+0.46 and its dusty ring nebula. The low surface gravities and dense winds of LBV stars are expected to yield a significant increase of the stellar radius - starting at near IR wavelengths - due to free-free processes. We have modeled these processes in a consistent manner, using the most recent version of the nonLTE model atmosphere code ISA-Wind of de Koter et al. $(1993,1997)$. This code treats the photosphere and stellar wind in a unified manner, i.e. it makes no artificial separation as is done in core-halo approaches. The adopted stellar parameters are $\mathrm{L}=3 \times 10^{5} \mathrm{~L}_{\odot}$, placing the star at $1800 \mathrm{pc}$, and a core radius of $45 R_{\odot}$. To fit the slope of the near IR continuum up to $\pm 10 \mu \mathrm{m}$ a mass loss of $1.3 \times 10^{5} \mathrm{M}_{\odot} / \mathrm{yr}$ was required. The terminal velocity was set at $100 \mathrm{~km} / \mathrm{sec}$, consistent with the width of $\mathrm{H} \alpha$ (see Voors et al., 1999, in preparation). This model yields a peak strength in $\mathrm{H} \alpha$ about 30 times continuum, in fair agreement with observations. The slope of the velocity law was set at $\beta=1$. Indeed, these parameters imply a wind that is so dense that at a thermalization optical depth $1 / \sqrt{3}$ at the center of the $V$ band (see de Koter et al., 1996) the flow velocity is already $6 \mathrm{~km} / \mathrm{sec}$. In other words, the stellar photosphere is formed in the wind - G79 shows a so-called 'pseudo photosphere'. The resulting stellar radius is $65 \mathrm{R}_{\odot}$, yielding a $\mathrm{T}_{\mathbf{e f f}}=16.6 \mathrm{kK}$. 
The observed and predicted energy distributions are presented in Figure 3. For the fits of the energy distribution we also used the ISO SWS spectrum from Morris et al. (1998, in preparation). The model fits both the optical and IR continuum very well, when assuming an extinction $\mathrm{E}(\mathrm{B}-\mathrm{V})=3.9$. Also the absorption at $10 \mu \mathrm{m}$, due to interstellar amorphous silicates, is in good agreement. Note the absorption at $3 \mu \mathrm{m}$ and the broad absorption between \pm 6 and $8 \mu \mathrm{m}$ are not reproduced by the model. These are likely due to water ices. At $4 \mu \mathrm{m}$ there is also absorption due to $\mathrm{CO}_{2}$ ices. These ices are probably located in the foreground radio source DR15.
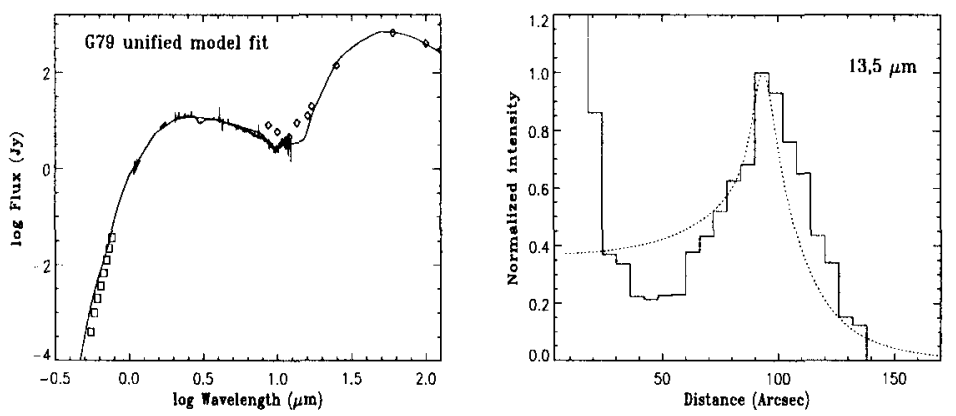

Fig. 3. Left: observed and predicted energy distribution from our model of the stellar atmosphere and the nebular dust shell. Right: Observed and fitted azimuthal integrated intensity profile for the G79.29+0.46 nebula.

The circumstellar material has been modeled assuming an optically thin dust shell, irradiated by the unified model. We have assumed the material to be composed of amorphous olivine grains. The grains are distributed in size from 0.01 to 0.1 micron following a power law distribution with index $\mathrm{m}=-3.5$. We require this model to fit both the energy distribution as well as the azimuthally integrated intensity profile as obtained from ISOCAM at continuum wavelengths $8.689,10.0,11.480,12.410,13.530,15.960$ and $17.0 \mu \mathrm{m}$. This last constraint yields a firm grip on the geometrical extension of the dust shell. We find an inner and outer radius of the dust of $2.48 \times 10^{18}$ and $1.10 \times 10^{19} \mathrm{~cm}$ respectively. The density distribution in the shell is assumed to be proportional to $\mathrm{r}^{-2}$. The dust temperatures range between $\mathrm{T}=61$ (large grains) and $64 \mathrm{~K}$ (small grains) at the inner boundary to $\mathrm{T}=38$ to $40 \mathrm{~K}$ at the maximum radius.

The model that fits the ISOCAM \& IRAS points best contains a dust mass of $0.15 \mathrm{M}_{\odot}$. Assuming a dust to gas ratio of 0.01 , the nebula contains $15 \mathrm{M}_{\odot}$ of material. The adopted stellar luminosity implies that G79 would have started out the main sequence as a $40 \mathrm{M}_{\odot}$ star. Evolutionairy tracks of Meynet et al. (1994) show that when such a star leaves the main sequence 
and starts its secular redward motion it first reaches $T_{\text {eff }}=20 \mathrm{kK}$ when it has lost $\pm 7 \mathrm{M}_{\odot}$. After a brief RSG phase, the star again increases in temperature as progressively deeper layers are exposed due to mass loss. As it now passes $\mathrm{T}_{\mathrm{eff}}=20 \mathrm{kK}$ on its blueward motion, the star has already lost $\pm 21 \mathrm{M}_{\odot}$. Near and during the RSG phase the star therefore looses some $14 \mathrm{M}_{\odot}$. Taking the derived shell mass at face value, this would imply G79 is most likely a postRSG star. The star will likely evolve into a WNL star.

The azimuthally integrated ISOCAM images also allow one to derive information on the geometry of the dust region. ISOCAM images show the dust region is fairly circular in shape. So, the dust may be in a spherical shell or in a more flattened disk-shape geometry, which we view pole on. Figure 3 shows the radial intensity profile at $13.5 \mu \mathrm{m}$. The observations are represented by the solid line. The dotted line gives the radial intensity of our spherical symmetric model. The ISOCAM resolution of $6 "$ at $15 \mu \mathrm{m}$ convolves the intensity of the central star out to several tens of arcsec. In the model this part of the intensity profile is excluded. The observations peak at 90 ", which corresponds to the distance of the inner radius of the shell, where the density, temperature and line of sight through the dust region are largest. At smaller distances from the core, the model intensity drops of to twice the emission of a radial column. If the observed emission drops below this limit - as is the case in G79 - this means that the dust distribution can not be spherical. The dust must be concentrated towards the equatorial plane, i.e. the geometry should be disk-shaped. We are currently investigating the precise geometrical distribution of the dust. Beyond 90", the intensity drops as the line of sight through the shell/disk decreases. The reasonable fit of our model to this part of the intensity profile suggests that in the inner radial segment of the dust shell (from 90 " to 140 ") the density distribution is reasonably well represented by the adopted $\mathrm{r}^{-2}$ dependence.

\section{References}

Cesarsky, C.J., Abergel, A., Agnese, P., et al. (1996): A\&A 315, L32

Clampin, M., Schulte-Ladbeck, R.E., Nota, A., Robberto, M., Paresce, F., Clayton, G.C. (1995): AJ 110, 251

de Koter, A., Schmutz, W., Lamers, H.J.G.L.M. (1993): A\&A 277, 561

de Koter, A., Lamers, H.J.G.L.M., Schmutz, W. (1996): A\&A 306, 501

de Koter, A., Heap, S.R., Hubeny, I. (1997): ApJ 477, 792

Higgs, L.A., Wendker, H.J., Landecker, T.L. (1994): A\&A 291, 295

Humphreys, R.M., Davidson, K. (1994): PASP 106, 1025

Hutsemékers, D. (1997): in "Luminous Blue Variables: Massive stars in Transition", eds. Nota and Lamers, ASP Conf. Ser. 120, p.316

Hutsemékers, D., Van Drom, E., Gosset, E., Melnick, J. (1994): A\&A 290, 906

Kessler, M.F., Steinz, J.A., Anderegg, M.E. et al. (1996): A\&A 315, L27

Lemke, D., Klaas, U., Abolins, J. et al. (1996): A\&A 315, L64

McGregor, P.J., Finlayson, K., Hyland, A.R., Joy, M., Harvey, P.M., Lester, D.F. (1988): ApJ 329, 874 
Meynet, G., Maeder, A., Schaller, D., Schaerer, D., Charbonnel, C. (1994): A\&AS 103, 97

Nota, A., Livio, M., Clampin, M. (1995): ApJ 448, 788

Nota, A., Clampin, M. (1997): in "Luminous Blue Variables: Massive stars in Transition", eds. Nota and Lamers, ASP Conf. Ser. 120, p.303

Robberto, M., Herbst, T.M (1998): ApJ 498, 400

Smith, L.J. (1994): in "Circumstellar media in late stages of stellar evolution", eds. Clegg et al., Cambridge University Press, p.64

Smith, L.J., Stroud, M.P., Esteban, C., Vilchez, J.M. (1997): MNRAS 290, 265

Trams, N.R., Waters, L.B.F.M., Voors, R.H.M. (1996): A\&A 315, L213

Viotti, R., Cassatella, A., Ponz, D., Thé, P.S. (1988): A\&A 190, 333

Voors, R.H.M., Waters, L.B.F.M., Trams, N.R., Käufl, H.U. (1997): A\&A 321, L21

Waters, L.B.F.M., Izumiura, H., Zaal, P.A., Geballe, T.R., Kester, D.J.M., Bontekoe, Tj.R. (1996): A\&A 313, 866

Waters, L.B.F.M., Morris, P.W., Voors, R.H.M., Lamers, H.J.G.L.M., Trams, N.R. (1998): Ap\&SS 255, 179

Wendker, H.J., Molthagen, K. (1998): Ap\&SS 255, 187

\section{Discussion}

G. Koenigsberger: In those cases where the nebula has a "horseshoe" shape, do you have an estimate for the density contrast required so that there is greatly reduced emission in certain portions of the shell?

N. Trams: The density contrast required would be similar to the contrast required to explain the empty central part of the nebula. This is rather high ( $1 / 10$ to $1 / 100$ ). The interesting thing in G 79 is that the highest density ISM is on the side of the gap in the nebula, therefore excluding the possibility of a nebula due to swept-up ISM.

M.-M. MacLow: Couldn't a thin shell seen in projection (e.g., with $\Delta \mathrm{R} / \mathrm{R}$ $\sim 12$, appropriate for an adiabatic shock) produce an observed circular shell with an apparently empty interior?

N. Trams: We have just started doing model calculations for the shell seen in G 79. The first calculations show that a thin spherical shell with a thickness of about $1 / 10$ of its radius still produces significant emission in the central area. 